\title{
Modelo educativo-pedagógico integrado de la Universidad de Otavalo, Ecuador
}

\author{
Cecilia J. Polaino*, Antonio de J. Romillo y Juan F. Muñoz \\ Universidad de Otavalo, Cdla Imbaya, Ave. De los Sarances s/n y Pendoneros, Cantón Otavalo, Provincia Imbabura, \\ Ecuador (correo-e: cpolaino@uotavalo.edu.ec; aromillo@uotavalo.edu.ec; fermuvi@hotmail.com).
}

Recibido Dic. 30, 2019; Aceptado Feb. 29, 2020; Versión final Abr. 15, 2020, Publicado Oct. 2020

\begin{abstract}
Resumen
El objetivo de este trabajo fue la determinación del modelo educativo-pedagógico integrado de la Universidad de Otavalo. Este modelo es producto del desarrollo de una investigación-acción participativa, basada en el análisis documental de los resultados de las fases críticas, cíclicas y en espiral del trabajo realizado en la propia universidad. El modelo integra cuatro elementos fundamentales:1) el modelo educativo y 2) el modelo pedagógico, con un mayor peso teórico conceptual, 3) el reglamento de régimen académico y 4) las estrategias metodológicas que posibilitan su aplicación práctica. Para cada uno de ellos se determinaron los componentes teóricos y prácticos correspondientes. Se identificaron los principales elementos y sus interrelaciones que resultaban decisivos para la toma de decisiones en la formación académica. Se concluye que el modelo educativo-pedagógico integrado está contribuyendo a importantes transformaciones cualitativas para alcanzar niveles superiores de calidad y desarrollo en la Universidad de Otavalo.
\end{abstract}

\section{Integrated educational-pedagogical model at the University of Otavalo, Ecuador}

\begin{abstract}
The objective of the present study was to develop an integrated educational pedagogical model for the University of Otavalo. The model was the product of the development of a participatory action research that was based on the documentary analysis of the critical, cyclical, and spiral phases of work performed at the University of Otavalo. The model integrates four fundamental elements: 1) the educational and 2) pedagogical models with a greater conceptual theoretical weight, 3) the regulation of academic regime, and 4) the methodological strategies that enable its practical application. The theoretical and practical components were determined for each fundamental element. The results identify the main elements and their interrelationships, which were decisive for decision-making in academic training. It is concluded that the integrated educationalpedagogical model contributes to important qualitative transformations to reach higher levels of quality and development at the University of Otavalo.
\end{abstract}

Keywords: educational model; pedagogical model; university; academic regime; methodological strategies 


\section{INTRODUCCIÓN}

La Ley Orgánica de Educación Superior de Ecuador (LOES), convoca a las universidades al desarrollo de actividades investigativas, de docencia y de vínculo con la sociedad dentro de una proyección educativa coherente y sistémica en busca de altos estándares de calidad, basados en un modelo educativo con identidad institucional propia. El análisis bibliográfico arroja diversas tendencias, concepciones y criterios en la elaboración de los modelos educativos y los modelos pedagógicos universitarios (León et al., 2016; ESPOL, 2016; Zaglul, 2016), muchos incluso denominan a estos últimos como académicos (Tünnermann, 2008); otros solo asumen el Modelo Educativo, aunque incluyen en el mismo, fundamentos, dimensiones, dominios 0 propuestas pedagógicas (Abreu, 2016; Trippl et al., 2015). Algunos se quedan en los fundamentos teóricos y otros los combinan con directrices para su aplicación práctica, pero por lo general todos responden a las concepciones filosóficas y pedagógicas asumidas por cada universidad para el logro de una gestión educativa de calidad (Dias, 2018; Agasisti, 2017; Harvey y Kosman, 2014; Voloshina y Shevchenko, 2014).

El pensamiento filosófico ha planteado, en diferentes épocas, proyecciones teóricas de cómo hacer corresponder la educación de las nuevas generaciones con el desarrollo de la sociedad, pasando desde posiciones reduccionistas de limitar la educación con el proceso de adquirir nuevos conocimientos, hasta entenderla como un sistema integrado de múltiples acciones educativas, como se concibe en la sociedad del conocimiento (Velasco, 2015). Los autores del presente trabajo consideran que en el mundo contemporáneo, las concepciones educativas de cualquier institución se deben basar en diversos criterios sobre cómo conducir la educación dentro de una sociedad con un alto desarrollo de la ciencia y la tecnología, sin olvidar los problemas ecológicos, económicos y sociales, el contexto en el que se desenvuelve la institución educativa y la participación activa de los actores principales en la construcción de sus modelos educativos y pedagógicos, ya que debe ser la propia comunidad universitaria la encargada de definir y dirigir sus propias necesidades, conflictos y soluciones.

La Universidad de Otavalo está enfrascada en profundas transformaciones cualitativas para alcanzar niveles superiores de calidad, que le han permitido su acreditación institucional y su desarrollo acelerado. Estas transformaciones han obligado a la actualización de los modelos educativo y pedagógico de la institución para lograr una formación integral, humana, científica y tecnológica, competencias profesionales con compromiso social en sus estudiantes. Para lograr dicha actualización se ha concebido un Modelo Educativo-Pedagógico Integrado que no se limite solamente a los fundamentos teóricos, sino que también aborde las directrices y procedimientos fundamentales para su correcta aplicación.

La investigación-acción participativa que permitió la construcción del Modelo se fundamentó en las concepciones desarrolladas por Lewin (1946), Stenhouse (1991), Fals y Rahman (1991), Elliot (1992), Anderson y Herr (1999), que junto a otros autores sentaron las bases de este tipo de investigación, se ubica en el paradigma crítico-propositivo, mediante la indagación introspectiva colectiva emprendida por los profesores y estudiantes participantes para mejorar sus propias prácticas educativas en el contexto de la institución. El desarrollo de la investigación-acción participativa, basada en el análisis documental de los resultados de las fases críticas, cíclicas y en espiral del trabajo realizado en la propia universidad, posibilitó identificar, mediante la utilización del método de análisis y síntesis, los principales elementos y su interrelación para la toma de decisiones en la formación académica, lo cual permitió conceptualizar el nuevo Modelo Educativo-Pedagógico Integrado de La Universidad, que se presenta en este trabajo.

En la construcción del modelo se siguieron los pasos siguientes: 1) aplicación de la Pirámide de Desarrollo Universitario como un novedoso modelo para la gestión (Romillo y Polaino, 2019), que posibilitó trazar el Plan Estratégico Desarrollo Institucional 2015-2020, que unido a la actualización de los Estatutos, sentaron las bases de la proyección filosófica institucional en la que se sustenta el modelo; 2) estudio documental sobre las tendencias más avanzadas de las concepciones de los modelos educativos y pedagógicos, así como de las normativas de la educación superior ecuatoriana; 3 ) análisis y síntesis de los requerimientos del proceso de la formación académica en la Universidad; 4) elaboración de una propuesta preliminar del modelo, por parte de una comisión de expertos con formación pedagógica, bajo la conducción del vicerrectorado; 5) análisis crítico de la propuesta por parte de las comisiones de carrera; 6) validación externa mediante una experto altamente calificado en la materia; 7) incorporación de las sugerencias y revisión del documento por la comisión legal; 8) socialización con profesores y estudiantes; 9) aprobación por el órgano colegiado superior; y 10) instrumentación del modelo a través de los colectivos de carrera, disciplina y asignaturas.

\section{METODOLOGİA}

La metodología de la investigación-acción participativa que permitió la construcción del Modelo, comenzó con la elaboración de la propuesta inicial por la comisión de expertos, la que fue enriquecida con la participación de los profesores y estudiantes de todas las carreras, mediante un proceso dialéctico, siguiendo una espiral de ciclos de planificación, acción, observación, reflexión. Se adoptó esta metodología, para 
mejorar los modelos educativos y pedagógicos hasta entonces vigentes en la universidad, mediante un método científico, en que los investigadores tienen un doble rol, de investigador y participante, combinando dos tipos de conocimientos: el teórico y la praxis en el contexto en que se desenvuelven, lo que posibilita además un proceso de aprendizaje que induce a las personas a teorizar acerca de sus prácticas mediante el análisis crítico de su quehacer, lo que conllevó a conceptualizar no solo los elementos teóricos del modelo, sino además a la actualización sistemática del reglamento de régimen académico de la universidad y de las estrategias metodológicas como instrumentos de su aplicación práctica, integrado todo en el mismo documento, que resulta una importante novedad que puede ser de utilidad en la construcción de modelos similares de otras universidades.

En el proceso de formación académica (grado y posgrado) intervienen los subprocesos que se señalan en la Figura 1; constituyen entradas al modelo: los estudiantes de grado, posgrado que desean cursar las carreras y programas que oferta la universidad; la Ley Orgánica de Educación Superior (LOES), normativas y reglamentos del Conejo de Educación Superior (CES), Consejo de Aseguramiento de la Calidad de la Educación Superior (CACES) y de la propia universidad; las necesidades investigativas y de vinculación con la sociedad que se presentan; los acuerdos, convenios y contratos con empresas, instituciones públicas, organizaciones no gubernamentales y otras universidades tanto locales, nacionales, como internacionales; los patrocinios, donaciones e ideas que provienen del contexto institucional; el personal que aspira a ingresar a la organización; los insumos y adquisiciones que requieren los procesos sustantivos, el funcionamiento de la universidad y los planes de mejora, entre otros (Polaino y Criollo, 2018).

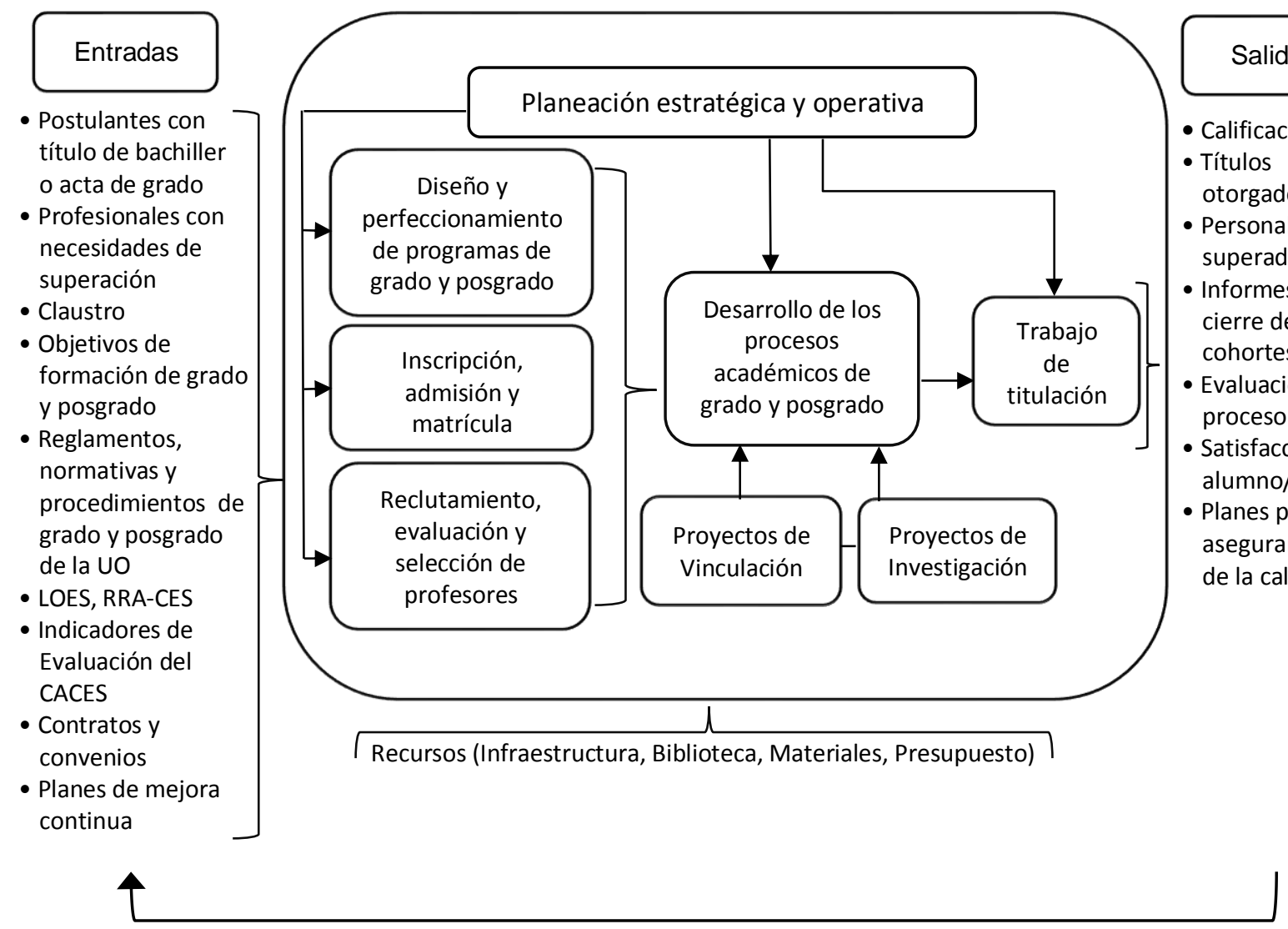

Fig. 1: Proceso de formación de grado y posgrado (reproducida con autorización de Polaino y Criollo, 2018)

A partir de las entradas y con el aseguramiento de los recursos necesarios, tienen lugar los procesos contenidos en el esquema de la figura 1, donde destaca el desarrollo de los procesos académicos (grado y posgrado) curriculares y extracurriculares, en el que juegan un papel fundamental los proyectos de investigación y de vinculación con la sociedad, que permiten integrar los procesos sustantivos de docenciainvestigación- extensión (este último en Ecuador se le denomina vinculación con la sociedad). La culminación de los procesos académicos, como también se observa en la figura, ocurre a través de un trabajo de integración de titulación en cualquiera de las modalidades establecidas en el currículo, todos los procesos son conducidos a través de los planes estratégicos-operativos y se enmarcan en el Modelo Educativo Pedagógico Integrado de la Universidad de Otavalo, que más abajo se expone. Como salidas están los egresados de las diferentes carreras, programas de posgrado, así como, todos los productos, servicios, publicaciones, ponencias, patentes, registros y resultados en sentido general, que aporta la universidad a la sociedad. 


\section{Modelo educativo-pedagógico integrado de la Universidad de Otavalo}

En la Figura 2 se muestra el esquema del Modelo Educativo-Pedagógico Integrado de la Universidad de Otavalo, que está enmarcado en un contexto institucional local, nacional e internacional, del cual provienen las entradas y al que tributan los productos, servicios y resultados de la universidad, en el que tiene prioridad el entorno más inmediato al que se debe fundamentalmente la institución: la región centro-norte del país y en particular Otavalo, con sus propias características interculturales.

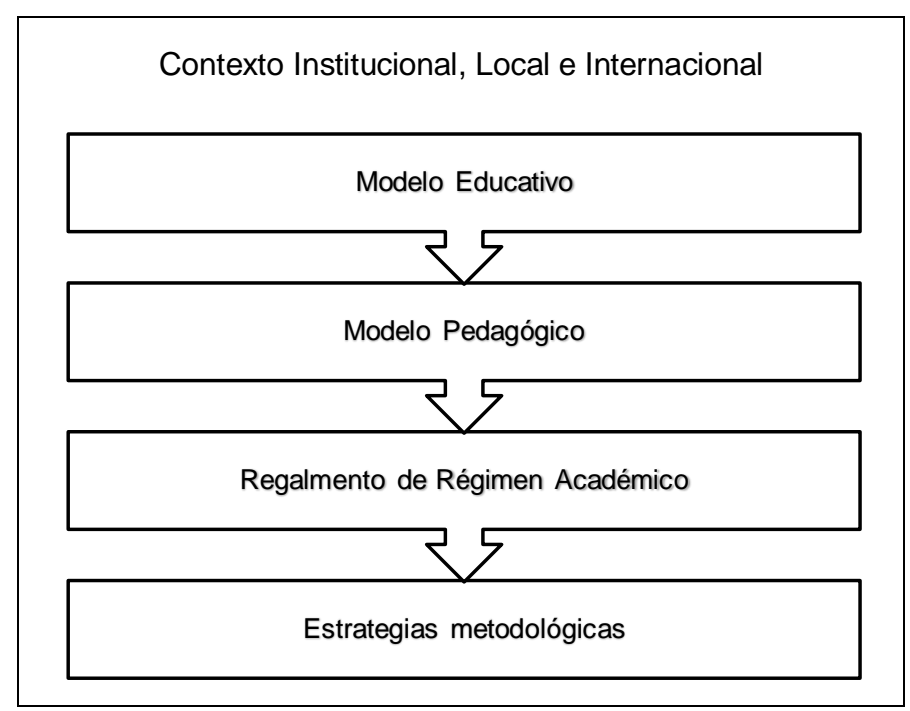

Fig. 2: Esquema del Modelo Educativo - Pedagógico Integrado de la Universidad de Otavalo

En el modelo integrado se diferencian los componentes con un peso teórico conceptual mayor, que son los modelos educativo y pedagógico, así como los componentes para su instrumentación práctica, que se derivan de los dos anteriores: el reglamento de régimen académico de la universidad y las estrategias metodológicas por carreras o por programas de posgrado.

\section{Modelo Educativo}

El Modelo Educativo de la Universidad de Otavalo se proyecta hacia el logro de altos estándares de calidad en la gestión universitaria, potenciando la investigación científica, la innovación tecnológica y empresarial, la vinculación con la sociedad, el desarrollo humanístico dentro de un contexto intercultural, así como el apoyo y fomento al emprendimiento y surgimiento de nuevas herramientas que promuevan el desarrollo social, económico y cultural de la región. El objetivo del Modelo Educativo es orientar los paradigmas educativos que profesa la Universidad de Otavalo que sivan como referencia para todas las funciones que cumple: formación, investigación, vinculación con la sociedad, gestión y emprendimiento, para el logro de su concepción educativa. El Modelo Educativo está sustentado en la historia, filosofía, principios fundacionales, valores compartidos y finalidades de la institución, así como, en los referentes teóricos educativos más avanzados; sus componentes se muestran en la Figura 3.

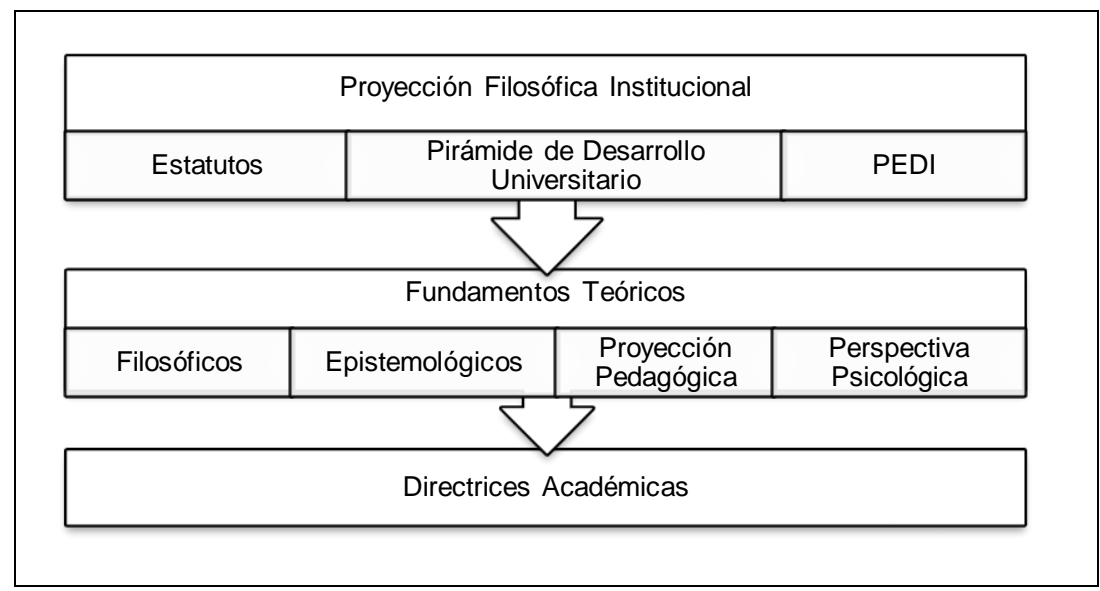

Fig. 3: Componentes del Modelo Educativo 


\section{Proyección filosófica institucional}

Las proyecciones filosóficas de la universidad han sido actualizadas frecuentemente, siendo la más recientes las establecidas en Plan Estratégico de Desarrollo Institucional correspondiente al periodo 2015-2020 (PEDI 2015-2020) y en la actualización de los Estatutos de la Universidad realizada en el año 2018, a partir de la modificación de la Ley Orgánica de la Educación Superior. Los principales elementos que caracterizan a la proyección filosófica institucional son los que se presentan a continuación. La Universidad de Otavalo a través de sus Estatutos, se rige por los principios de autonomía responsable, cogobierno, igualdad de oportunidades, calidad, pertinencia, integralidad y autodeterminación para la producción de pensamiento y de conocimiento en el marco del diálogo de saberes, pensamiento universal, producción científica y tecnológica global (Ley 0, 2018, Art. 12), inclusión, entendida como la construcción de capacidades y políticas que garanticen el acceso ciudadano en igualdad de oportunidades y la vigencia plena del espíritu democrático. Al mismo tiempo, en las disposiciones generales de los Estatutos, la Universidad garantiza la existencia de las organizaciones gremiales en su seno, las que tendrán sus propios estatutos que guardarán concordancia con la ley y las normas institucionales.

El Plan Estratégico de Desarrollo Institucional correspondiente al periodo 2015- 2020, se sustenta en la Pirámide del Desarrollo Universitario, como novedoso modelo para la gestión (Romillo y Polaino, 2019; Acosta et al., 2017), que comprende cuatro componentes fundamentales, estos son: 1) las áreas de resultado clave, 2) las restricciones de desarrollo, 3) el contexto institucional; y 4) la dirección estratégica de la organización, que enmarca y abarca todos los elementos esenciales que guían la gestión de la institución de educación superior y sus procesos hacia los planos superiores deseados del desarrollo universitario. Las pocas, pero esenciales áreas de resultados, donde se encuentran los factores críticos de éxito, que moverán a la institución en la dirección estratégica correcta y en las que se concentran los objetivos estratégicos de la universidad son: claustro de profesores; formación académica; investigación; vinculación con la sociedad y aseguramiento logístico.

\section{Fundamentos teóricos del Modelo Educativo}

El Modelo Educativo se ha elaborado sobre la base de un conjunto de referentes teóricos de orden filosófico, epistemológico, dimensión pedagógica y perspectiva psicológica, que se resumen a continuación.

Fundamentos filosóficos. El Modelo Educativo de la Universidad de Otavalo presta su atención en el desarrollo humanístico, articulado con el sistema de conocimientos científico-tecnológicos, el pensamiento transdisciplinario y multidisciplinario, por encima del conocimiento especializado y fragmentado. El proceso de enseñanza-aprendizaje está centrado en el aprendizaje, orientado a la búsqueda de la verdad y a la solución de problemas reales de la profesión y la vida.

Forman parte de la proyección filosófica del Modelo Educativo los elementos siguientes: contribuir a la identificación y solución de los problemas del país, mediante la formación integral, continua de profesionales, la investigación y la vinculación con la sociedad; por su origen y el contexto donde se desarrolla, posee una orientación hacia la interculturalidad, antropología y el desarrollo sustentable; es una institución abierta a las corrientes del pensamiento universal, sin proselitismo político, ni religioso; busca la excelencia a través de la práctica de la cultura de calidad en todos sus actos; la formación consciente, participativa, crítica con rigor científico, que comprenda los derechos fundamentales del ser humano y la sociedad; el cultivo de valores éticos y cívicos; el fomento de los derechos humanos con profunda conciencia ciudadana; la búsqueda de la verdad y formación de personas de honor, libres y disciplinadas; el mantenimiento de las bases históricas de la identidad nacional, para incrementar el orgullo de lo que somos, y proyectarnos hacia el futuro; la conservación, defensa, cuidado del medio ambiente, el uso racional de los recursos naturales; y, la práctica de los valores tradicionales de orden, disciplina, lealtad, justicia, gratitud y en el contexto de la responsabilidad, la honestidad a toda prueba, el autocontrol, la creatividad, el espíritu democrático, la solidaridad y la solución de los problemas mediante el diálogo y la razón, enfatizando en los valores compartidos declarados en su visión: capacidad creativa, responsabilidad, entrega emotiva, avance sustentable y relaciones solidarias.

Los futuros profesionales graduados por la Universidad de Otavalo tendrán competencias para integrarse a la sociedad del conocimiento, ser proactivos, emprendedores, satisfaciendo las necesidades personales y sociales, promoviendo la sustentabilidad; exigencias que serán expresadas en los perfiles profesionales de cada carrera.

Fundamentos epistemológicos. En la proyección epistemológica (gnoseológica), se asume el conocimiento como la interacción entre la actividad práctica y el pensamiento sistematizado que permite comprender, explicar, interpretar y mejorar su realidad. A su vez, coincidiendo con Collado (2016) plantea que el conocimiento es un producto de la actividad del ser humano en el trabajo, en relación activa con la 
naturaleza y la sociedad, por lo que la educación se apoya en la metodología transdisciplinar y en el principio de biomímesis con el propósito de reforzar los lazos entre la educación y la sostenibilidad, mediante encuentros pacíficos y sostenibles entre las diferentes culturas y civilizaciones que coexisten. Estos planteamientos se justifican con las siguientes adopciones teóricas: el conocimiento se concibe como proceso en construcción y transformación permanente en el que el estudiante es responsable de su propio aprendizaje; desarrollo de las habilidades de buscar, seleccionar, analizar, comunicar y evaluar la información, asumiendo un papel activo en la construcción de su propio aprendizaje.

Los estudiantes se consideran como elementos activos de sus aprendizajes, a través de actividades que les permitan exponer e intercambiar ideas, aportaciones, opiniones y experiencias con sus compañeros hacia el desarrollo de habilidades y destrezas en el mundo de las competencias; los conocimientos científicos se organizan en forma secuencial, a través de diversas actividades derivadas de proyectos, estudio de casos y solución a problemas de la sociedad, de forma coherente e integrada a fin de situar al estudiante en contacto con su entorno, lo que se sustenta en las dos ideas centrales de la epistemología del sur (Sousa, 2018) que son la ecología de saberes y la traducción intercultural; $y$, los contenidos son seleccionados, organizados y tratados considerándolos como información útil que se comparte con el estudiante y no como datos que deben ser memorizados y repetidos en forma mecánica e independiente de la realidad.

Dimensión pedagógica. La Pedagogía como Ciencia de la Educación ha desarrollado distintos enfoques teóricos de cómo conducir la actividad educativa: el tecnológico-estructural, el hermenéutico-interpretativo y los enfoques críticos o socio-críticos. Existen diversas posiciones en cuanto a concebir el acto educativo desde una perspectiva crítica (Cabaluz-Ducasse, 2016), que significa poner al estudiante constantemente en situaciones de aprendizaje que implican: reflexión, valoración y crítica de lo que se estudia desde diferentes puntos de vista; búsqueda de soluciones a los problemas reales de la vida, de propuestas para la acción y transformación; y el desarrollo humanístico, articulación de conocimientos, habilidades y valores en función de las competencias de actuación para la vida.

La dimensión pedagógica del Modelo Educativo de la Universidad de Otavalo, proyecta desarrollar el proceso educativo con énfasis en las concepciones de la "Pedagogía Crítica", que potencian el protagonismo de los estudiantes en la solución de problemas de la vida social, económica y tecnológica; así como, un currículo flexible, pertinente, que atiende necesidades de la sociedad y de los estudiantes, y se actualiza con los últimos avances de la ciencia y la tecnología. De igual forma se considera dentro de la propuesta pedagógica del modelo educativo, la formación de profesionales basada en los cuatro saberes fundamentales planteados por Delors: saber aprender, saber hacer, saber convivir, y, saber ser (Fortoul - Ollivier, 2016).

Perspectiva psicológica. Se parte del criterio de integrar diversas teorías y metodologías del aprendizaje en función de lograr una participación activa y significativa de los estudiantes. Se potencia el aprendizaje cognitivista (aprendizaje por descubrimiento y significativo a partir de conocimientos previos) y constructivista (rol activo del individuo en el aprendizaje), que considera la aplicación y sistematización de procesos diversos como la comprensión de textos, comparar, hacer resúmenes, elaborar mapas que reflejan la interpretación del material de estudio, conceptualizar con ideas propias, resolver problemas, debatir puntos de vista, generalizar bases teóricas y construir- producir nuevas soluciones con creatividad.

\section{Directrices académicas}

El Modelo Educativo de la Universidad de Otavalo, a partir de sus concepciones filosóficas institucionales y de los fundamentos teóricos, orienta el desarrollo de la academia a través de un conjunto de directrices entre las cuales se pueden mencionar las siguientes: la formación integral de profesionales comprometidos con el desarrollo económico y social del país; formación centrada en el aprendizaje y en la educación continua; estrategias de aprendizaje que promueven el logro de los cuatro saberes; desarrollo de habilidades del pensamiento y aprendizaje basado en problemas; grupos colaborativos en el desarrollo de habilidades del trabajo en equipo; combinación dinámica de teoría-práctica; aplicación de diversos enfoques pedagógicos orientados a la formación profesional y humanística, a partir de metodologías activas de aprendizaje sustentadas en la práctica de valores, la interculturalidad y equidad de género; integración entre la docencia, investigación y vinculación con la sociedad a lo largo del proceso de formación profesional; diseño curricular a partir de un sistema de competencias profesionales sobre una base estructural de unidades integradoras del contenido; empleo de las tecnologías de la información y la comunicación (TIC) (Sosa y Manzuoli, 2019) con un enfoque participativo e interactivo apoyado en estas herramientas (Bidarra y Rusman, 2017); promoción del desarrollo sustentable de la sociedad y protección del ambiente como objetivo permanente de todo el quehacer profesional; desarrollo de un sistema de valores humanos de carácter general y específico en cada una de las profesiones; evaluación del proceso de aprendizaje en función del desempeño y resultados; $y$, fomento al desarrollo profesional de los docentes. 


\section{Modelo Pedagógico}

El Modelo Pedagógico de la Universidad Otavalo es el referente teórico - metodológico que orienta el desarrollo del proceso enseñanza y aprendizaje, a partir de la definición de los fundamentos filosóficos, epistemológicos, pedagógicos y psicológicos, definidos en el Modelo Educativo; existe plena correspondencia entre ambos modelos y, constituyen herramientas que se construyen a partir de la realidad económica y social de la provincia de Imbabura, y en particular, del entorno de Otavalo. De igual forma se tuvo en cuenta la base conceptual y legal de la Constitución de la República de Ecuador y de la Ley Orgánica de Educación Superior del Ecuador. El objetivo del Modelo Pedagógico es orientar el desarrollo del proceso enseñanza y aprendizaje en la Universidad de Otavalo, a partir de los fundamentos filosóficos, epistemológicos, pedagógicos y psicológicos, definidos en el Modelo Educativo institucional, en función de alcanzar los estándares de calidad que plantean los organismos rectores de la educación superior y que se ha trazado la propia institución. El conjunto de componentes teóricos y prácticos que caracterizan el Modelo Pedagógico de la Universidad se puede representar de forma esquemática como se muestra en la Figura 4.

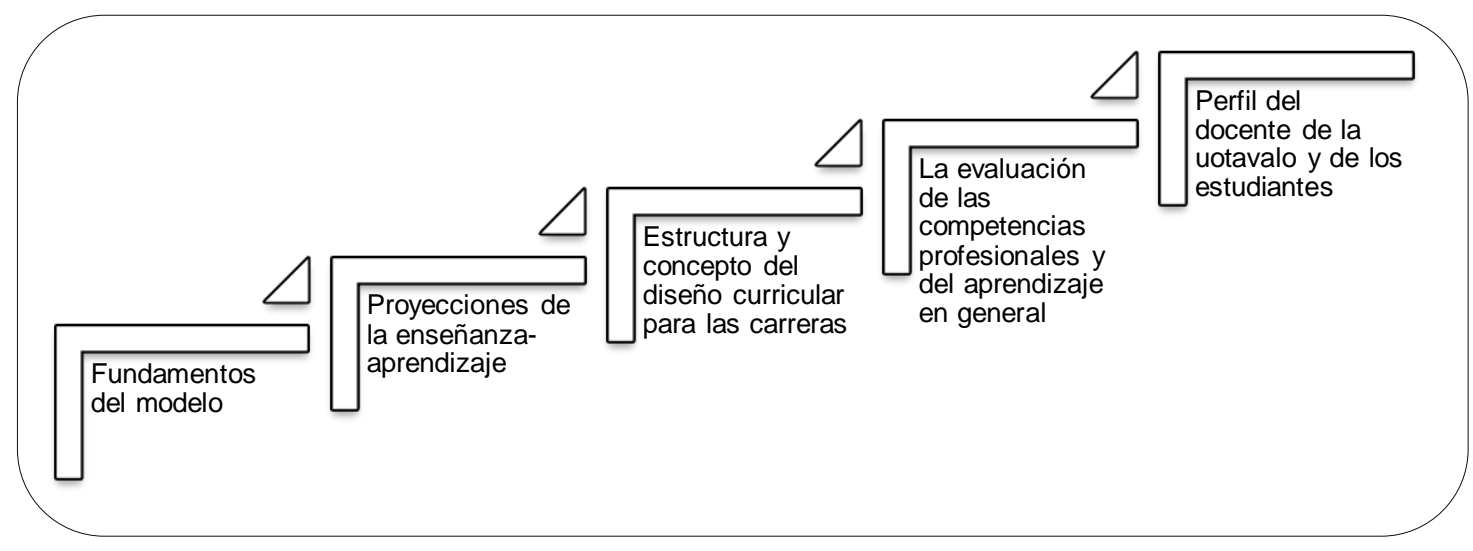

Fig.4 Componentes del Modelo Pedagógico

\section{Fundamentos del Modelo Pedagógico}

El Modelo Pedagógico, a partir de la base teórica del modelo educativo, se ha elaborado sobre los fundamentos pedagógicos del proceso docente educativo, los paradigmas del aprendizaje y la concepción del proceso de formación. Teniendo en cuenta la proyección pedagógica definida en el Modelo Educativo, las actividades académicas se articulan dentro de un diseño curricular que estructura coherentemente las experiencias de aprendizaje a desarrollar en función de competencias profesionales, para que los estudiantes participen, desde el aprendizaje, en su transformación con la solución de problemas, la investigación, la innovación y producción.

El Modelo Pedagógico interpreta y aplica diversos enfoques teóricos y metodológicos del aprendizaje, considerando fundamentalmente: el aprendizaje basado en la interacción social de L. Vigostky (Helou y Newsome, 2018); el aprendizaje basado en la acumulación de experiencias de J. Piaget (Ginsburg y Opper, 2016 y McLeod, 2018); el aprendizaje por descubrimiento de J. Bruner, ya que mediante una metodología constructivista, el alumno puede estar inmerso en experiencias significativas, asimilando así nueva información (Millar y Eden, 2011); y el aprendizaje significativo de D. P. Ausubel (Agra et al., 2019).), destacan la significación del enfoque histórico-cultural de Vigotsky y sus aportes fundamentales en cuanto a la enseñanza y el desarrollo, su concepción sobre el aprendizaje y la categoría situación social del desarrollo para el tratamiento de las relaciones interdisciplinarias.

En las bases teóricas del modelo pedagógico de la Universidad de Otavalo se define como paradigmas que el aprendizaje es un proceso natural y necesidad del ser humano; es un proceso social (interactividad constante del grupo estudiantil); es activo, no pasivo (investigación, solución de problemas, producción y aporte de criterios); puede ser tanto lineal como no lineal (procesamiento simultáneo de varias ideas y puntos de vistas); solución de problemas con varias alternativas, integrado y contextualizado (articulación de los conocimientos y habilidades de las diferentes disciplinas de estudio y vinculación con la vida); basado en un modelo que se fortalece en contacto con las habilidades y la cultura del estudiante (aprendizaje basado en experiencias y la construcción del conocimiento en el entorno de la interculturalidad); se evalúa según los productos del proceso (forma en que se cumplen las tareas y la solución de problemas reales, tanto por cada estudiante como por el grupo); y, la evaluación del aprendizaje exige la presentación y defensa de productos acreditables de los resultados del mismo (sobre la base del estudio de casos, solución de problemas reales, ejecución de proyectos integradores e investigaciones), e iniciativas estratégicas diseñadas para mejorar la experiencia de aprendizaje de los estudiantes (Ayres, 2018). 
Concepción del proceso de formación. Ante el constante desarrollo en la sociedad del tercer milenio, las instituciones de educación superior orientan sus modelos educativos en función de lograr repuestas eficientes a los requerimientos cambiantes de la ciencia, tecnología, ecología, economía y la sociedad en general. En este sentido el Modelo Pedagógico sustenta su accionar universitario dentro de las concepciones siguientes: la investigación es el eje articulador de todo el proceso de formación de los futuros profesionales; el vínculo con la sociedad se produce de forma constante a través de los procesos de investigación, la realización de prácticas pre-profesionales, la realización de proyectos integradores de base curricular y de proyectos de innovación tecnológica, empresarial y social; el diseño curricular se estructura a partir de un sistema de competencias profesionales sobre una base estructural de unidades integradoras del contenido y de las experiencias de aprendizaje a desarrollar; el empleo de las tecnologías de la información y la comunicación en el proceso de enseñanza-aprendizaje constituye una vía esencial para ampliar el volumen de información a procesar por los estudiantes, promover un mayor dinamismo en la actividad de estudio y favorecer una educación interactiva y personalizada; el fomento de emprendimientos, con el apoyo de las asignaturas, proyectos de investigación y de vinculación con la sociedad, generan una cultura empresarial que a la vez, potencian el liderazgo y el desarrollo sustentable de la sociedad; la protección del ambiente como objetivo permanente de todo el quehacer profesional; $\mathrm{y}$, el desarrollo de un sistema de valores humanos de carácter general y específico en cada una de las carreras, en función de formar un profesional competente integral.

\section{Proyecciones de la enseñanza y el aprendizaje}

El proceso de enseñanza y aprendizaje se estructura y dinamiza en el Modelo Pedagógico, a través de los ejes articuladores y de los métodos de aprendizaje. Se consideran como ejes articuladores los siguientes: la formación basada en competencias profesionales; la integración de la formación científica, tecnológica y humanística; los proyectos integradores de investigación por etapas de formación; la gestión del aprendizaje con el apoyo de las TIC; y, los emprendimientos con apoyo de la gestión académica universitaria y tecnológica; que se describen a continuación.

Formación basada en competencias profesionales. Las competencias profesionales orientan el nivel de desempeño a lograr, de forma progresiva, en los estudiantes; constituyen estándares de calidad de los resultados finales del aprendizaje. Se conceptualizan las competencias profesionales como: conjunto de cualidades que caracterizan comportamientos humanos generalizadores, dentro de una perspectiva integradora y compleja del pensamiento y modo de actuación para la solución de problemas sociales. Al asumir esta definición de competencia, se le da una connotación de referente orientador y articulador del sistema de conocimientos, habilidades y desarrollo humano que se proyecta para las diferentes áreas de estudio. En el contexto de los programas académicos la competencia se puede definir, entonces, como: "La capacidad de un profesional para tomar decisiones, con base en los conocimientos, habilidades y actitudes asociadas a la profesión, para solucionar los problemas complejos que se presentan en el campo de su actividad profesional" (Galdeano y Valiente, 2010); su desarrollo y evaluación en los procesos de formación académica se realizan fundamentalmente a través de los currículo, como más adelante explica.

Integración de la formación científica, tecnológica y humanística. El proceso de enseñanza y los aprendizajes se desarrollan con una adecuada articulación de la formación científica y tecnológica, que se requiere en las diferentes carreras, con la formación humanística; es decir, una proyección holística del ser humano que incorpora a su modo de actuación una sólida preparación cultural y un sistema de valores integrados a las bases teórico- prácticas del quehacer profesional. La formación cultural- humanística se desarrolla por dos vías: dentro de un conjunto de materias de estudio comunes a todas las carreras de la Universidad y como eje transversal desde el contenido y metodologías de todas las asignaturas y módulos de contenidos, bien sean de formación básica o profesional para la carrera. Los diseños micros curriculares proyectan este enfoque holístico: ciencia-tecnología y formación cultural-humanística en el sistema de unidades de aprendizaje, con sus bases conceptuales, problemas, casos, proyectos y las diversas tareas de estudio.

Proyectos integradores de investigación por etapas de formación. Para dar solución a los problemas de gran dimensión que requieren la articulación de los métodos de la investigación científica y los métodos propios de diferentes ciencias, se requiere un instrumento articulador que planifique y organice el sistema de tareas que conduce a la solución de los mismos; ese instrumento es el proyecto integrador de investigación que articula los contenidos aislados, fraccionados en asignaturas y unidades temáticas, en función de obtener un producto final que evidencie resultados significativos del aprendizaje.

Los proyectos integradores de investigación se realizan dentro de la estructura curricular y se planifican en función de las competencias a desarrollar, pasando inicialmente por acciones de procesamiento de información científica-técnica, por aplicaciones situacionales concretas y por el enfrentamiento a problemas que requieren una alta integración y sistematización de los contenidos de estudios. La evaluación se realiza de forma frecuente, estableciendo metas o resultados parciales que deben verificarse para generar la 
retroalimentación del proceso de desarrollo del proyecto. Por último, se convoca a la evaluación final donde el estudiante presenta la memoria científica del proceso investigativo realizado y sus resultados, tanto de forma escrita como mediante una defensa oral ante un equipo de docentes que represente las principales áreas del saber referidas en el proyecto.

Empleo de las tecnologías de la información y la comunicación, en la gestión del aprendizaje. Las TIC favorecen la aplicación de nuevas prácticas educativas y evaluación de los aprendizajes para el desarrollo de las competencias profesionales, la interactividad, el trabajo colaborativo, la autonomía y las habilidades interpersonales y de comunicación de los estudiantes, considerando que el uso de la tecnología es una herramienta para solucionar los problemas, automatizar los procesos, mejorar la calidad de vida, mejorar los procesos de aprendizaje que, conjuntamente con las metodologías pedagógicas y técnicas didácticas, permitan la generación del conocimiento. Los diversos recursos tecnológicos que posee la Universidad de Otavalo tales como: pizarras digitales interactivas, proyectores, computadoras, internet y el sistema para la gestión del aprendizaje, disponible en todas las aulas, constituyen una excelente oportunidad para favorecer los procesos de enseñanza y aprendizaje.

Emprendimientos con apoyo de la gestión académica universitaria y tecnológica. El emprendimiento es una actividad compleja que converge en factores sociales, personales, cognitivos, económicos, culturales y políticos, que son predictores en las iniciativas del emprendimiento (Durán-Aponte y Arias-Gómez, 2015); generalmente los emprendimientos se realizan de forma empírica sin conocer el comportamiento del mercado, sus normas o reglamentos, entre otros factores; es por esto que los emprendimientos surgen como una posibilidad de abrir campos, que permiten fortalecer el desarrollo socio económico de los sectores a los que puede acceder la universidad. Es necesario considerar dentro del plan de estudios la promoción de una cultura que permita facilitar los emprendimientos, que no opaque las iniciativas académicas estudiantiles y más bien, contribuya a desarrollarlas, fortalecerlas y ejecutarlas, con el fin de que los proyectos y propuestas de los estudiantes sean exitosos y sustentables en el tiempo, convirtiéndose en promotores y gestores del desarrollo económico. La Universidad de Otavalo, en la medida de lo posible, aportará con la gestión académica y tecnológica la promoción de los proyectos mejor enfocados en el territorio, comenzando desde las tutorías que propenderán en incentivar las ideas y emprendimientos generados por los estudiantes hasta la presentación, ejecución, monitoreo y seguimiento de los mismos.

Métodos de aprendizaje. Los ejes articuladores se concretan a través de los métodos de aprendizaje, que guían el accionar metodológico para el desarrollo de las competencias profesionales; estos son: aprendizaje basado en problemas, aprendizaje basado en casos de la práctica social y el aprendizaje colaborativo. La idea esencial del aprendizaje basado en problemas $(A B P)$ es presentar el contenido objeto de estudio a partir de un problema real de la vida y en el proceso de análisis, buscar un nuevo conocimiento que permita resolverlo; en la medida en que el estudiante se enfrente sistemáticamente a variadas situaciones que generen la aplicación de este proceso, desarrollará las competencias necesarias para su profesión. El aprendizaje basado en casos de práctica social o estudio de casos $(A B C)$ demanda acciones investigativas que promueven la reflexión, comparaciones y conclusiones, así como, la confrontación de puntos de vistas, lo que constituye un componente esencial en la formación de las competencias profesionales. El aprendizaje colaborativo, se enfoca a que los alumnos sean capaces de interactuar socialmente con otros estudiantes y con los profesores, lo que implica contribuir para lograr un objetivo compartido (Barkley, 2014); esto requiere la disposición y actitud activa por parte de los estudiantes y el rol del profesor como asesor o guía; de igual forma, se potencia la integración entre alumnos de distintas culturas, religiones y costumbres debidos al carácter multicultural que cada vez más adquiere la sociedad. Los tres métodos de aprendizaje que se utilizarán a través del trabajo práctico, tanto en escenarios reales como virtuales, realizando actividades productivas, de servicios o sociales, constituye un eslabón fundamental en la consolidación del contenido objeto de estudio y en la consecución y desarrollo de las competencias de actuación para la profesión y de la vida en general.

\section{Estructura y concepción del diseño curricular para las carreras}

El Modelo Pedagógico proyecta un diseño curricular basado en competencias profesionales de carácter genéricas y específicas, donde se articulan los contenidos de las diversas materias de estudio en función de alcanzar desempeños integradores que reflejan un conjunto de cualidades y capacidades, para resolver los problemas de la ciencia, la tecnología y la sociedad en general. Dentro del diseño curricular de cada carrera se define un perfil profesional que orienta el sistema de competencias a desarrollar y una malla curricular que articula las diversas experiencias de aprendizaje a desarrollar, en función de las competencias. Los conocimientos disciplinares, interdisciplinares, transdisciplinares, profesionales, investigativos, de saberes integrales y de comunicación se organiza en asignaturas, cursos o sus equivalentes y las asignatura responde a una o más disciplinas de estudio en función de contribuir a la formación de una competencia, articulando actividades teóricas y prácticas, en aulas, laboratorios, talleres y trabajo de campo o en escenarios reales. 
La estructura curricular garantiza el proceso de formación y de aprendizaje y comprende las unidades de organización curricular y los campos de formación del currículo. Las unidades de organización curricular ordenan las asignaturas, cursos o sus equivalentes, acorde con el nivel de aprendizaje en cada período académico, articulando los conocimientos de modo progresivo e integrador, a lo largo de la carrera o programa. Los campos de formación clasifican los conocimientos disciplinares, profesionales, investigativos, de saberes integrales y de comunicación, necesarios para desarrollar el perfil profesional y académico del estudiante al final de la carrera o programa y se organizan los conocimientos en función de sus propósitos, objetos y problemas de estudio de la carrera o programa. Por lo tanto, la estructura curricular evidencia la consistencia, coherencia y correspondencia interna entre: el perfil de ingreso, las relaciones entre los conocimientos y saberes del conjunto de las asignaturas, cursos o sus equivalentes y el perfil de egreso, aportando al desarrollo y fortalecimiento de las capacidades integrales de los futuros profesionales.

Las unidades de organización curricular en las carreras de grado se estructuran de la manera siguiente: 1) unidad básica: que introduce al estudiante en el aprendizaje de las ciencias y disciplinas que sustentan la carrera, sus metodologías e instrumentos, así como en la contextualización de los estudios profesionales; 2) unidad profesional: que está orientada al conocimiento del campo de estudio y las áreas de actuación de la carrera, a través de la integración de las teorías correspondientes y de la práctica pre profesional; y 3) unidad de titulación: que incluye: asignaturas, cursos o sus equivalentes, que permiten la validación académica de los conocimientos, habilidades y desempeños adquiridos en la carrera para la resolución de problemas, dilemas o desafíos de una profesión; su resultado final fundamental es: a) el desarrollo de un trabajo de titulación, basado en procesos de investigación e intervención o, b) la preparación y aprobación de un examen de grado de carácter complexivo.

La planificación curricular incluye la elaboración de los programas de estudio de las asignaturas y proyectos integradores de investigación, con la inclusión de productos y tareas de aprendizaje a desarrollar; así como, la estrategia para evaluar los logros de desempeño. Para el diseño de los programas de estudio se requiere confeccionar, inicialmente, redes lógicas del contenido y de los resultados del aprendizaje que se esperan en cada área del conocimiento, que permiten proyectar de forma lógica y coherente los conocimientos y habilidades a desarrollar en cada asignatura. En la planificación del micro currículo se consideran los siguientes elementos: objetivos de la asignatura; competencias genéricas y específicas; actividades de aprendizaje; metodología; evaluación; resultados del aprendizaje y evidencias para el portafolio académico, bibliografía básica y complementaria; y, cronograma del desarrollo de actividades, todo lo cual se plasma en el sílabo de la asignatura. Para la aplicación de lo señalado se toma como eje los resultados de aprendizaje que contribuyen al cumplimiento de los objetivos y perfiles de egreso de la carrera.

El sistema de clases y tutorías. Los contenidos de estudio y las tareas de aprendizaje en función de las competencias profesionales, se potencian desde el sistema de clases, que desde una perspectiva universitaria considera: conferencias orientadoras del contenido de estudio, los productos del aprendizaje a obtener, la bibliografía a consultar y los procedimientos de la evaluación; clases prácticas donde se sistematizan habilidades a través de la realización de ejercicios, del estudio de casos y de la solución de problemas reales; clases de laboratorios donde se comprueban leyes y se aplican bases teóricas; clases debates o fórum que invitan a la reflexión, la confrontación y sistematización de ideas y procesos de estudio; clases en escenarios reales y virtuales, que posibilitan profundizar conocimientos en condiciones de un proceso profesional concreto; y clases de evaluación que permiten valorar los resultados del aprendizaje con los desempeños integradores proyectados. Las tutorías en proyectos de cursos, de investigación, de titulación e incluso en la realización de las tareas de curso, son muy importantes, pues el profesor ejerce la función de guía y el estudiante consulta de manera presencial, tutorías digitales y otros.

\section{La evaluación de las competencias profesionales y del aprendizaje en general}

Para evaluar las competencias profesionales de forma progresiva, en la medida que el estudiante avanza en la proyección curricular, se requiere de las valoraciones colegiadas del conjunto de docentes de la carrera, siguiendo las exigencias de estándares de calidad e indicadores operativos que se esperan alcanzar. Los estándares de calidad expresan la profundidad, el grado de efectividad en la aplicación de conocimientos, habilidades y el desarrollo humano que demandan las competencias; y los indicadores operativos son evidencias concretas de los resultados del aprendizaje que demandan los estándares. La evaluación del aprendizaje desde todas las materias de estudio y en especial de los proyectos integradores de investigación constituyen la vía esencial para valorar en qué medida se van logrando, de forma progresiva, las competencias de actuación profesional, tanto genéricas como específicas; es concebida por el Modelo Pedagógico, como un proceso sistemático, frecuente e integrador del desempeño, que demuestran los estudiantes lo que demanda la aplicación de diversas técnicas donde se jerarquiza: las preguntas en clases y en la actividad de tutoría; búsqueda y procesamiento de información científica y cultural; elaboración de diversos productos escritos como ponencias, ensayos, monografías y otros; participación en foros-debates; 
solución de problemas reales; realización de pruebas escritas y orales; defensa de proyectos en clase, investigación y estudios de casos. Este proceso debe demandar conocimientos y modos de actuación integradores en complejidad creciente, en la medida que el estudiante avanza en el proceso de formación profesional.

\section{Perfil del docente y del estudiante para aplicar el Modelo Pedagógico}

Para aplicar eficientemente el Modelo Pedagógico se necesita docentes con alta preparación profesional, científica, cultural y humanística que respondan a los requerimientos del modelo; y a la vez, estudiantes capaces de interactuar de forma exitosa dentro de las exigencias que se proyectan en el mismo. Los docentes deben poseer títulos de cuarto nivel en correspondencia con el área del conocimiento con la cual laborarán y demostrar las competencias profesionales siguientes: valores y liderazgo para conducir el proceso educativo universitario con firmeza y calidez humana, respeto a la interculturalidad; expresa una cultura de investigación y de actualización científica-técnica en la profesión y materias que desarrolla en la Universidad de Otavalo; aplica diversos métodos y técnicas para promover el aprendizaje significativo en los estudiantes; emplea las tecnologías de la información y la comunicación en el desarrollo del aprendizaje y en la gestión académica; y, demuestra dominio del Modelo Educativo-Pedagógico Integrado, sus fundamentos conceptuales, directrices y proyecciones metodológicas.

De igual manera, el estudiante de la Universidad de Otavalo es un bachiller con titulación nacional o extranjera que posee las competencias básicas siguientes: demuestra interés por la profesión seleccionada; se comunica de forma fluida y coherente; interpreta textos y la realidad del entorno natural y social; interpreta y resuelve problemas con la aplicación de un pensamiento lógico matemático y conceptos de las ciencias básicas; y, demuestra una personalidad estable, con equilibrio emocional y respeto a las normas sociales.

\section{Reglamento de Régimen Académico}

Para la instrumentación acertada del Modelo Educativo-Pedagógico Integrado, la Universidad de Otavalo ha concebido como parte del mismo su Reglamento de Régimen Académico, que regula y orienta el quehacer académico de la Universidad de Otavalo en sus diversos niveles de formación. El reglamento tiene como objetivos los siguientes: a) garantizar una formación de alta calidad que propenda a la excelencia y pertinencia, mediante su articulación con las necesidades de la transformación y participación social, b) regular la gestión académica formativa en todos los niveles y modalidades de aprendizaje, con miras a fortalecer la formación académica y profesional, la investigación y el servicio comunitario; c) promover la diversidad, integralidad y flexibilidad de los itinerarios académicos, entendiendo a éstos como la secuencia de niveles y contenidos en el aprendizaje y la investigación; d) articular la formación académica y profesional, la investigación científica, tecnológica y social; y, el vínculo con la sociedad, en un marco de calidad, innovación y pertinencia; e) contribuir a la formación del talento humano y al desarrollo de profesionales y ciudadanos críticos, creativos, deliberativos y éticos, que desarrollen conocimientos científicos, tecnológicos y humanísticos, comprometiéndose con las transformaciones de los entornos sociales y naturales; y, respetando la interculturalidad, igualdad de género y demás derechos constitucionales; f) desarrollar una educación centrada en los sujetos educativos, promoviendo el desarrollo de contextos pedagógicocurriculares interactivos, creativos y de construcción innovadora del conocimiento y los saberes; g) impulsar el conocimiento de carácter multi, inter y transdisciplinario en la formación de grado y posgrado, la investigación y el vínculo con la sociedad; $h$ ) desarrollar la educación superior bajo la perspectiva del bien público social, aportando a la democratización del conocimiento para la garantía de derechos y la reducción de inequidades; i) promover la movilidad de profesores e investigadores y estudiantes para su integración en la dinámica del conocimiento a nivel nacional e internacional; y j) propiciar la participación en redes académicas y de investigaciones regionales, nacionales e internacionales, para el desarrollo de procesos de producción del conocimiento y los aprendizajes profesionales.

Los principales componentes que conforman el reglamento de régimen académico son los siguientes: ámbito y objetivos; organización del proceso de aprendizaje (estructura curricular, trabajo metodológico, planificación curricular y planificación académica; evaluación del aprendizaje, aprobación y reformas de las carreras y programas de posgrado, modalidades de estudio o aprendizaje y aprendizaje de personas con discapacidad); interculturalidad; itinerarios académicos, reconocimientos u homologaciones y titulación; investigación (plan anual de investigación; propiedad intelectual y derechos de autor; resultados científicos y su difusión; investigación científica estudiantil); servicio comunitario y vinculación con la sociedad (educación continua y formación docente, practicas pre profesionales y pasantías); estructuras institucionales de la universidad; y redes académicas. El reglamento de régimen académico de la Universidad se concibe como un instrumento práctico dialéctico, susceptible a actualizaciones sistemáticas en correspondencia con la espiral introspectiva de ciclos de planificación, acción, observación, reflexión propios de la investigación acción participativa que se realiza. 


\section{Estratégias metodológicas por carreras o programas de posgrado}

Las estrategias metodológicas por carreras o programas de posgrado se concretan semestralmente en el Plan de Trabajo Metodológico, brindan orientaciones más específica sobre cómo desarrollar la enseñanza y los aprendizajes de acuerdo con la lógica interna de cada ciencia, se elaboran sobre la base de las proyecciones estratégicas de la carrera o programa de posgrado, el análisis de la eficiencia de la oferta académica y el informe sobre los resultados del trabajo docente educativo del semestre anterior, cerrándose con ello el ciclo de las orientaciones teóricas y prácticas del modelo educativo-pedagógico integrado de la Universidad de Otavalo.

Las acciones que contemplan están dirigidas a mejorar en la práctica cotidiana el desarrollo de los procesos sustantivos universitarios: docencia, investigación y vinculación con la sociedad, a través del aseguramiento de la calidad de las clases, prácticas, tutorías, evaluación del aprendizaje, contenidos de las asignaturas, proyectos de investigación y de vinculación con la sociedad, mallas curriculares, programas de estudio, eficiencia académica, sistema de competencias profesionales, capacitación de los profesores y otros procedimientos educativos, metodológicos y organizativos que se consideren pertinentes.

\section{DISCUSIÓN}

A través de una investigación acción se logró construir un modelo educativo-pedagógico integrado para la Universidad de Otavalo, que consta de cuatro elementos fundamentales, los modelos educativo y pedagógico con un mayor peso teórico conceptual, así como el reglamento de régimen académico y las estrategias metodológicas que posibilitan su aplicación práctica; para cada uno de ellos se determinaron los componentes teóricos y prácticos correspondientes por carreras o por programas de posgrado. Tanto el modelo en sí, como la metodología de investigación acción utilizada para su construcción, presentan novedades importantes, que pueden ser de utilidad en la construcción de modelos similares de otras universidades.

El modelo educativo pedagógico integrado se proyecta hacia el logro de altos estándares de calidad, potenciando la investigación científica, la innovación tecnológica y empresarial, la vinculación con la sociedad, así como el desarrollo humanístico dentro de un contexto intercultural; pretende la formación integral centrada en el aprendizaje de profesionales comprometidos con el desarrollo económico y social del país; el proceso de enseñanza - aprendizaje se estructura y dinamiza a través de los métodos de aprendizaje y los ejes articuladores: la formación basada en competencias profesionales, la integración de la formación científica, tecnológica y humanística, los proyectos integradores de investigación por etapas de formación, la gestión del aprendizaje con el apoyo de las TIC y, los emprendimientos con apoyo de la gestión académica universitaria y tecnológica.

El modelo educativo pedagógico Integrado está siendo referencia obligatoria de las principales acciones y reglamentaciones de las funciones sustantivas universitarias que se están actualizando, así como en su primer año de aplicación se aprecian mejoras en los indicadores en la eficiencia académica y en la satisfacción de los estudiantes con su formación, por lo que se puede concluir que el mismo está contribuyendo a las importantes transformaciones cualitativas para alcanzar niveles superiores de calidad y desarrollo de la Universidad de Otavalo.

\section{CONCLUSIONES}

De acuerdo al trabajo presentado y a los resultados obtenidos, se pueden plantear las siguientes conclusiones principales:

1.- La investigación acción desarrollada posibilitó construir el Modelo Educativo-Pedagógico Integrado de la Universidad de Otavalo

2.- El modelo integra cuatro elementos fundamentales, los modelos educativo y pedagógico con un mayor peso teórico conceptual, así como, el reglamento de régimen académico y las estrategias metodológicas que posibilitan su aplicación práctica

3.- El Modelo Educativo Pedagógico integrado está contribuyendo a las importantes transformaciones para alcanzar niveles superiores de calidad y desarrollo de la Universidad de Otavalo

4.- Tanto el modelo en sí, como la metodología de investigación acción utilizada para su construcción, presentan novedades importantes, que pueden ser de utilidad en la construcción de modelos similares de otras universidades 


\section{REFERENCIAS}

Abreu, O., Naranjo, M.E., Rhea, B.S. y Gallegos, M.C, Modelo Didáctico para la Facultad de Ciencias Administrativas y Económicas de la Universidad Técnica del Norte en Ecuador, https://doi: 10.4067/S0718-50062016000400002, Revista Formación Universitaria Vol. 9(4), 3-10 (2016).

Acosta, L.A, Becerra F.A. y Jaramillo D., Sistema de Información Estratégica para la Gestión Universitaria en la Universidad de Otavalo (Ecuador), https://dx.doi.org/10.4067/S0718-50062017000200011, Revista Formación Universitaria, 10(2), 103-112 (2017).

Agasisti, T., Management of Higher Education Institutions and the Evaluation of their Efficiency and Performance, https://doi: 10.1080/13583883.2017.1336250, Tertiary Education and Management, 23:3, 187-190, (2017)

Agra, G., Formiga, N.S, Oliveira, P.S, Costa, M.M.L., Fernandes, M.G.M., y Nóbrega, M., Analysis of the concept of Meaningful Learning in light of the Ausubel's Theory, https://doi.org/10.1590/0034-7167-2017-0691, Rev Bras Enferm, 72(1):248-55, (2019).

Anderson, G.L. y Herr, K. The new paradigm wars. Is there room for rigorous practitioner knowledge in schools and universities? Educational Researcher, 28(5), 12-21, (1999).

Ayres, R.L., "Impact assessment in higher education: a strategic view from the UK", https://doi.org/10.1108/ILS-06-20170064,Information and Learning Sciences, Vol. 119 No. 1/2, pp. 94-100., (2018)

Barkley, E., El aprendizaje colaborativo en la universidad: referentes y práctica. REDU, Revista de Docencia Universitaria, Vol. 12 (4), 281-302 ISSN: 1887-4592, Barcelona, España, (2014).

Bidarra, J. y Rusman, E., Towards a pedagogical model for science education: bridging educational contexts through a blended learning approach, https://doi: 10.1080/02680513.2016.1265442, Open Learning: The Journal of Open, Distance and e-Learning, 32:1, 6-20, (2017).

Cabaluz-Ducasse, J.F., Pedagogías críticas latinoamericanas y filosofía de la liberación: potencialidades de un diálogo teórico-político. Educación y Educadores, 19 (1), 67-88, ISSN 0123-1294, (2016).

Collado, J., Una perspectiva transdisciplinar y biomimética de la educación para la ciudadanía mundial, Educere, 20 (65), 113-129, (2016).

Dias, E. Strategic Management of Educational Institutions Enabling Transformation to Excellent Education Innovative Centers in India. International Journal of Management, Technology, and Social Sciences (IJMTS), https://doi: https://doi.org/10.5281/zenodo.1189056, 3(1), 25-36, (2018).

Durán-Aponte, E. y Arias-Gómez, D., Intención emprendedora en estudiantes universitarios: integración de factores cognitivos y socio-personales. Revista Colombiana de Ciencias Sociales, 6(2), 320-340, (2015).

Elliot, J., Action research for educational change: Developing teachers and teaching. ISBN 0-335-09689-1, Philadelphia: Open University Press, Milton Keynes, (1992).

ESPOL, Reforma Curricular 2016 Proyecto Educativo Innovador, Escuela Superior Politécnica del Litoral, Ecuador, (2016).

Fals, O. y Rahman, MD., ANISUR, Acción y conocimiento: Rompiendo el monopolio con la IAP, Bogotá, (1991).

Fortoul - Ollivier, M.B., The different type of knowledge and its relevance in the subjects' training, https://doi: https://doi.org/10.26457/recein.v13i47.106, Revista del Centro de Investigación de la Universidad La Salle, 12 46), 171196, (2016).

Galdeano, C. y Valiente, A., Competencias profesionales, Educación química [online], 21(1), 28-32, (2010).

Ginsburg H.P. y Opper, S., Piaget's Theory of Intellectual Development (3rd Edition), ISBN-10: 013675158X; ISBN-13: 978-0136751588, International Psychotherapy Institute e-Book, (2016).

Harvey, M y Kosman, B.E., A model for higher education policy review: the case study of an assessment policy, https://doi: 10.1080/1360080X.2013.861051, Journal of Higher Education Policy and Management, 36:1, 88-98, (2014)

Helou, M.M. y Newsome, L.K., Application of Lev Vygotsky's Sociocultural Approach to Foster Students Understanding and Learning Performance, https://doi.org/10.22158/jecs.v2n4p347, Journal of Education and Culture Studies, 2(4), 347353, (2018).

León, M., Ortega, C.E. y Estrada, V., Fundamentos del Modelo Educativo para la enseñanza online de la Universidad de Especialidades Espíritu Santo, Ecuador. Revista científica ECOCIENCIAS, 3 (5), ISSN: 1390-9320, (2016).

Lewin, K., Action research and minority problems"; Journal of Social Issues 2 (4), 34-46, (1946).

McLeod, S.A., Jean Piaget's theory of cognitive development. Simply Psychology, (2018).

Millar, J. y Eden, S., Primarily play: engaging primary learners through play. ETFO, (2011).

Polaino, C y Criollo, J., Sistema de Gestión de Calidad para el Proceso de Formación de Posgrado de la Universidad de Otavalo. Revista Sarance No.38, ISSN 1390-9207, 141-162, Ecuador, (2018).

Romillo, A y Polaino, C., Aplicación del Modelo de Gestión Pirámide del Desarrollo Universitario en la Universidad de Otavalo, Ecuador, https://doi.org/10.4067/S0718-50062019000100003,Formación universitaria, 12(1), 3-12, (2019). 
Sosa, O., y Manzuoli, C., Models for the pedagogical integration of information and communication technologies: a literature review, https://doi.org/10.1590/s0104-40362018002701720, Ensaio: Avaliação e Políticas Públicas em Educação, 27(102), 129-156, (2019).

Sousa, B., Epistemología del Sur: un pensamiento alternativo de alternativas políticas, https://doi.org/10.24215/2346898Xe032, Geograficando 14 (1), e032, (2018).

Stenhouse, L., Research and curriculum development, ISBN 10:8471122200, Published by Ediciones, Madrid: Morata, S.L., (1991).

Trippl, M., Sinozic, T., y Lawton H., The Role of Universities in Regional Development: Conceptual Models and Policy Institutions in the UK, Sweden and Austria, https://doi: 10.1080/09654313.2015.1052782, European Planning Studies, 23:9, 1722-1740, (2015)

Tünnermann B., Modelos educativos y académicos. ISBN: 978-99924-79-35-3, Nicaragua: Editorial Hispamer, (2008).

Velasco, R. Modelo educativo para la sociedad del conocimiento, https://doi: 10.13140/RG.2.2.18510.92488, Revista Universidad Nacional Pedagógica de México, 19, (2015).

Voloshina, V. y Shevchenko, T., The strategic management tools for higher education institutions. https://doi 10.17721/1728-2667.2014/155-2/11, Екоһотіка. 2(155), ISSN 1728-2667, 2(155):55-60, 2014

Zaglul, J.A., EARTH University educational model: perspective on agricultural educational models for the twenty-first century, https://doi: 10.1080/21553769.2016.1193826, Frontiers in Life Science, 9:3, 173-176, (2016). 